\title{
Bringing heterogeneity to the CMS software framework
}

\author{
Andrea Bocci ${ }^{1}$, David Dagenhart ${ }^{2}$, Vincenzo Innocente $^{1}$, Christopher Jones ${ }^{2}$, Matti \\ Kortelainen $^{2, *}$, Felice Pantaleo ${ }^{1}$, and Marco Rovere ${ }^{1}$ \\ ${ }^{1}$ CERN, Geneva, Switzerland \\ ${ }^{2}$ Fermi National Accelerator Laboratory, Batavia, IL, USA
}

\begin{abstract}
The advent of computing resources with co-processors, for example Graphics Processing Units (GPU) or Field-Programmable Gate Arrays (FPGA), for use cases like the CMS High-Level Trigger (HLT) or data processing at leadership-class supercomputers imposes challenges for the current data processing frameworks. These challenges include developing a model for algorithms to offload their computations on the co-processors as well as keeping the traditional CPU busy doing other work. The CMS data processing framework, CMSSW, implements multithreading using the Intel Threading Building Blocks (TBB) library, that utilizes tasks as concurrent units of work. In this paper we will discuss a generic mechanism to interact effectively with non-CPU resources that has been implemented in CMSSW. In addition, configuring such a heterogeneous system is challenging. In CMSSW an application is configured with a configuration file written in the Python language. The algorithm types are part of the configuration. The challenge therefore is to unify the CPU and co-processor settings while allowing their implementations to be separate. We will explain how we solved these challenges while minimizing the necessary changes to the CMSSW framework. We will also discuss on a concrete example how algorithms would offload work to NVIDIA GPUs using directly the CUDA API.
\end{abstract}

\section{Introduction}

Co-processors or computing accelerators like graphics processing units (GPU) or fieldprogrammable gate arrays (FPGA) are becoming more and more popular to keep the cost and power consumption of computing centers under control. For example, GPUs are used in many leading supercomputers, are being used in a trigger farm by ALICE [1-5], and are being considered in trigger farms for the Run 3 data taking of the Large Hadron Collider [6] in CMS [7, 8] and LHCb [9, 10]. The CMS data processing framework (CMSSW) [11-15] implements multi-threading using the Intel Threading Building Blocks (TBB) [16] library utilizing tasks as units of concurrent work. While in principle non-CPU resources could be interacted with in the TBB tasks directly in a straightforward way, the non-CPU APIs typically imply blocking the calling thread. Such blocking would lead to under-utilizing the CPU.

In this paper we describe generic mechanisms to interact with non-CPU resources effectively from the TBB tasks (Section 2), and to configure CPU and non-CPU algorithms in a

\footnotetext{
*e-mail: matti@fnal.gov
} 
unified way that works well together with the rest of the CMS computing infrastructure (Section 3). As a first step to gain experience, we have explored various ways for how algorithms could offload work to NVIDIA GPUs with CUDA [17]. Section 4 describes a pattern that we have found most effective so far, and has also the least impact on the rest of the CMSSW framework.

\section{Concurrent CPU and non-CPU processing}

When computations are offloaded to non-CPU resources, the CPU program needs to eventually know when the offloaded work is finished. The simplest way to perform this synchronization is to introduce a blocking wait on the CPU thread ${ }^{1}$, i.e. the CPU thread waits for the completion of the offloaded work. The CPU thread can wait either by busy waiting or sleeping. The downside of the former approach is that the CPU core is unable to do other work, implying that such waits should be short at best, while the downside of the latter approach is that the latency from the work completion to the CPU thread resuming work is longer than in the former approach. CMS data processing applications typically have some work that could be done concurrently with the offloaded work, and therefore the busy waiting would clearly lead to wasting CPU resources.

In the case of CMS applications, the thread-sleeping approach also has a subtle downside. The number of available CPU cores is decided externally to the application, and may be less than the total number of logical CPU cores of the compute node. In addition, we can not assume that the compute node would enforce the limit on the number of CPU cores the CMS application is allowed to use, instead the CMS application should act as a good citizen and keep at most the allowed number of CPU cores busy on the average. With CPU-only work good CPU utilization can be achieved simply by initializing the TBB thread pool to use the same number of threads as the number of cores, and letting the TBB task scheduler keep the threads busy. In this way all the CPU cores are kept utilized as long as there are enough tasks to fill the threads, without a risk of using additional CPU cores. Offloading computational work and synchronizing the CPU thread by sleeping to wait for the offloaded work to finish would lead to under-utilization of the CPU cores. In principle the application could be configured to use more threads than allowed CPU cores, but then the ratio of threads to cores would become a tunable parameter that would depend for example on the exact application type, the CPU performance, the offloaded-to-resource performance, and the data being processed. In order to avoid introducing such an additional tunable parameter, we chose to develop a generic mechanism that allows the CPU thread to run other TBB tasks while the offloaded work is being run elsewhere

The basic idea of the External Worker concept is to replace the blocking waits with a callback-style solution. Traditionally the algorithms scheduled by the CMSSW framework (called modules) have one function that is called by the framework for each event. The exact function name depends on the module type ${ }^{2}$, for the simplicity in the following only the producer module case is described. The concept itself, however, is general and works similarly with filter and analyzer modules as well. It could be further noted that the External Worker concept resembles the async_node in the TBB Flow Graph library [16].

The traditional produce() member function is split into two stages: acquire and produce. First, the framework calls an acquire() member function, that can only read event data products, and should launch the offloaded work. The acquire() function is given

\footnotetext{
${ }^{1}$ E.g. in CUDA cudaDeviceSynchronize (), cudaStreamSynchronize(), cudaEventSynchronize().

${ }^{2}$ Analyzer modules are only allowed read event data products and have a member function analyze(), producer modules can also insert new data products and have a member function produce(), and filter modules can also decide whether a trigger path should continue or stop execution and have a member function filter()
} 
a reference-counted holder object (edm: :WaitingTaskWithArenaHolder) that holds the TBB task that will make the framework call the produce () function. The holder object is intended to be notified upon completion of the offloaded work. Internally the holder decreases the reference count, and once the count reaches zero, the contained TBB task is enqueued to the task arena the holder also holds a pointer to. Thanks to the explicit use of the task arena the holder can be given to non-TBB threads to be signaled. The holder is also capable of delivering exceptions. See Section 4.1 on how this mechanism can be used with CUDA.

\section{Unified configuration for CPU and non-CPU algorithms}

CMS uses a hash of the application configuration to segregate data from different workflows. The simplest approach to configure jobs using GPUs would be to create a configuration different from a CPU-only job. In this approach, however, the data from a single dataset processed with CPU-only and with GPU resources would have different hashes, and therefore would be treated as different datasets. Such a feature would significantly restrict the flexibility of the CMS data processing workflow management system, which consists of a global pool of jobs that can, in principle, run at any site. To preserve the flexibility of processing parts of a dataset on any architecture, the configuration hash must be the same for all architectures.

We wanted to be able to keep CPU and non-CPU algorithms separate to enable an evolutionary migration path. For example, in order to introduce non-CPU algorithms, the current, working and validated, CPU algorithms can be left untouched. In addition, the natural work division may differ for different hardware architectures. It could also happen that some nonCPU architectures are in conflict in a way that prevents dynamically loading their libraries into the same application. On the other hand, we do not want to preclude having CPU and non-CPU algorithm in the same module either.

The CMSSW framework already tracks the input data of each module event by event. We decided to use the same provenance tracking mechanism to store also information about the choice of technology. This information enables us to inspect afterwards the architecture on which a given event was processed.

Based on the aforementioned goals, we developed the SwitchProducer concept in the CMSSW configuration, depicted in Figure 1. The SwitchProducer allows specifying multiple modules that are associated to the same module label ${ }^{3}$. The modules for different cases can be either totally different modules, or differently configured instances of the same module. Thus all possibilities are specified in the part of the configuration that affects the hash computation. The mechanism makes the choice between the cases at runtime on the worker node based on available technologies. The mechanism relies on the CMSSW's module scheduling logic of consumer modules dictating which producer modules are run. For example in the case of Figure 1, if the worker node has a GPU, only the hits@gpu module is run to produce the input for seeds module. If, on the other hand, the worker node does not have a GPU, both hits@cpu and clusters modules are run.

It should be noted that the SwitchProducer requires that the producer modules of all the cases produce exactly the same data product types (hits@cpu and hits@gpu in in Figure 1). This constraint ensures that the choice by the SwitchProducer is transparent to all consumer modules (e.g. seeds in Figure 1).

\footnotetext{
${ }^{3}$ In CMSSW each module must have a label that is unique within a process. The event data products produced by the module are associated to the module label.
} 

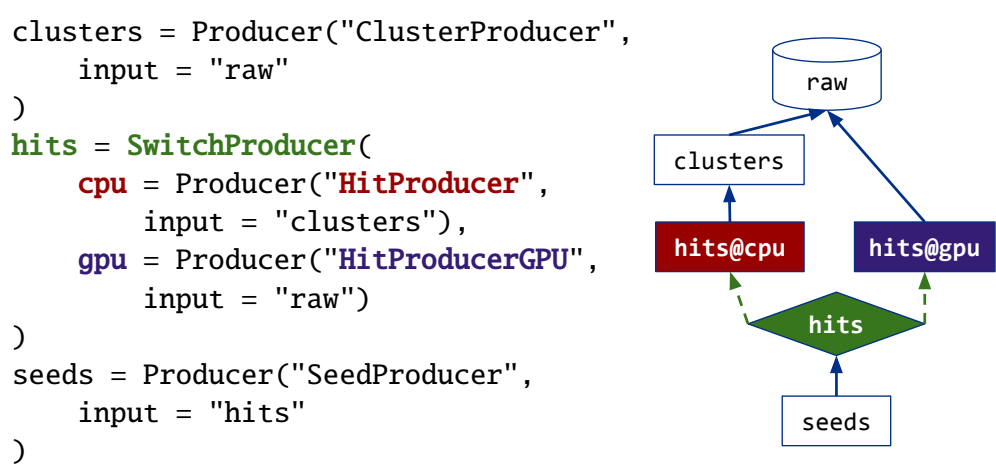

Figure 1. A configuration fragment showing an example of how the SwitchProducer would look like (left), and a data dependence graph corresponding the configuration (right). On the CPU case, the HitProducer depends on a data product clusters, whereas on the GPU case, the HitProducerGPU takes directly raw as an input. The SwitchProducer decides at runtime on the worker node which of the two producers should be used.

\section{Pattern to interact with CUDA runtime}

Based on the external worker (Section 2) and SwitchProducer (Section 3) concepts we developed tools and a pattern to interact with the CUDA runtime from CMSSW modules. The pattern is described as follows. We wanted the CPU to be able to do other work while the GPU is running an algorithm. This asynchronous execution is described in Section 4.1. We wanted to minimize data movements between the CPU and the GPU. This goal required the ability to share resources like GPU memory or a CUDA stream between modules, which is described in Section 4.2. A mechanism to transfer data only when necessary is then described in Section 4.3. The design of the tools should also be extendable to multiple non-CPU device types, and be able to make use of multiple devices per type.

Much of the interaction with the CMSSW framework is done by a wrapper template cms : : cuda: : Product $<\mathrm{T}>$ for data products (of type $\mathrm{T}$, which itself can be partly or fully in the GPU memory), and a helper object cms : : cuda : : ScopedContext ${ }^{4}$ that is intended to be used in the body of the module's acquire () and produce () functions.

The pattern has similar functionality as CUDA graphs [17], that is a directed acyclic graph of memory transfers, kernel launches, and host functions, but at a higher level. In theory CUDA graphs could be used in the background, but preliminary investigations indicate that the current implementation of CUDA graphs is too restrictive for our usage pattern.

\subsection{Asynchronous execution}

In order to avoid the CPU waiting for GPU work to finish only the asynchronous CUDA runtime API calls may be used during event processing. Essentially this constraint means memory transfers and memset calls, because the kernel launches are asynchronous by construction. The asynchronous API calls require the use of CUDA streams. Work items queued in a single CUDA stream are executed serially, but concurrently with respect to work in other CUDA streams.

\footnotetext{
${ }^{4}$ In reality the class has a couple of variants, but for the discussion in this paper grouping them into one is sufficient.
} 
The Product $<\mathrm{T}>$ and ScopedContext tools were developed such that each parallel branch in the module $\mathrm{DAG}^{5}$ automatically gets its own CUDA stream. With such an approach the available concurrency is maximally expressed to the CUDA runtime, which can then schedule work as it sees best.

In addition, possible synchronization points need to be carefully avoided. These synchronization points include for example memory allocations with the CUDA runtime API, explicit synchronization calls, and calls to assert () in kernel code. The simplest way to avoid dynamic memory allocations through the CUDA runtime API during event processing would be to allocate the necessary device and pinned host memory for each module at the beginning of the job. This approach has, however, several drawbacks. Most importantly, it would lead to allocation of much more memory than is actually needed at any given time to cover all possible cases: 1) need to allocate memory for all concurrent events even though not all of them will be processed by the same module at the same time; 2) not all modules will be running concurrently because of data dependencies; and 3) the allocated memory would have to be large enough to cover the largest need of the processed events, and typically there are large variations between events.

To address all these drawbacks, we decided to use a memory pool for both the device and the pinned host memory for the memory allocations done on the host. At the time of writing these memory pools are based on the CachingDeviceAllocator from the CUB library [18]. While this memory pool allocates its memory during the event processing, essentially by caching the allocations, the cost of the API calls gets amortized.

It should be noted that only modules that need to synchronize the GPU and CPU for some CUDA stream, for example to transfer some data from GPU to CPU, need to use the External Worker mechanism (Section 2) instead of an explicit synchronization call ${ }^{6}$. Modules that only queue asynchronous GPU work can call the CUDA runtime API directly, in a way that resembles the streaming_node in the TBB Flow Graph library [16].

The proper signaling of work completion is handled by the ScopedContext: the WaitingTaskWithArenaHolder must be given to the constructor of the ScopedContext, and the destructor of ScopedContext queues a callback function into its CUDA stream with cudaStreamAddCallback () to which the WaitingTaskWithArenaHolder is passed. The callback function then notifies the WaitingTaskWithArenaHolder, and in case of errors, creates an exception object to be propagated.

\subsection{Sharing of resources between modules}

A chain of modules with producer-consumer relationships on the data in GPU memory run most efficiently if they agree at least on running their work on the same device. Furthermore, it would be beneficial for a linear chain of work to be queued into the same CUDA stream, and in case of branches in the DAG, let the CUDA runtime to deal with the synchronization between the branches.

The GPU data product wrapper Product $<\mathrm{T}>$ holds the device ID, the CUDA stream where the producing work was queued into, and a CUDA event to mark the completion of the asynchronous processing in case that did not finish by the time the module's produce() function ended. A module that queues more GPU work with Product $<\mathrm{T}>$ as an input constructs ScopedContext with the Product $<\mathrm{T}>$ as an argument. The ScopedContext then sets the current device based on the input product, and re-uses the CUDA stream from the input product if the module is the first one to ask it from the Product $<\mathrm{T}>$. If another module

\footnotetext{
${ }^{5}$ The modules form a directed acyclic graph (DAG) by their data dependencies.

${ }^{6}$ See footnote 1
} 
re-used the CUDA stream first, the ScopedContext creates a new CUDA stream and uses that.

The only way for a consumer to obtain $\mathrm{T}$ from Product $<\mathrm{T}>$ is via the ScopedContext. Upon request by the module, the ScopedContext checks whether the Product $<\mathrm{T}>$ uses the same CUDA stream as the ScopedContext was constructed with. If it does, the T object can be returned immediately, because the sequential nature of CUDA stream ensures the proper synchronization. In case the CUDA streams are different, the availability of $\mathrm{T}$ is checked via the CUDA event. If the CUDA event indicates that the asynchronous work producing $\mathrm{T}$ has completed, the $\mathrm{T}$ can again be returned safely. If the work is still incomplete, the ScopedContext introduces a wait on its CUDA stream on the CUDA event of the Product $<\mathrm{T}>$ by calling cudaStreamWaitEvent () before returning the $\mathrm{T}$ object.

\subsection{Minimization of data movements}

The CMSSW framework runs a producer module only if some other module consumes the output data product of the producer module. We can make use of this behavior to minimize the data transfers from GPU to CPU by adding additional, specific modules that only queue the data transfers. This way the transfers are avoided if no other module asks for a CPU copy of data in GPU memory, but the capability to do the transfer exists in case a module asking for such a copy is added into the configuration.

This approach works well together with the SwitchProducer mechanism in the configuration (Section 3), and the way data in GPU memory is passed from one module to another (Section 4.2). In practice the user is expected to use the SwitchProducer to choose between the CPU module, and the module that transfers the GPU data back to CPU, and leave all the dependent GPU modules to be run by the framework.

\section{Summary}

This paper described the generic building blocks we have developed for CMSSW that can be used to continue the exploration of using non-CPU resources for CMS data processing. We are exploring the performance characteristics of the described pattern for using CUDA from the data processing modules. An example of the achieved performance on a real-world application can be found in [8].

\section{Acknowledgements}

This document was prepared by the CMS Collaboration using the resources of the Fermi National Accelerator Laboratory (Fermilab), U.S. Department of Energy, Office of Science, HEP User Facility. Fermilab is managed by Fermi Research Alliance, LLC (FRA), acting under Contract No. DE-AC02-07CH11359.

\section{References}

[1] ALICE Collaboration, JINST 3 S08002 (2008)

[2] D. Rohr et al, J. Phys.: Conf. Series 396, 012044 (2012)

[3] D. Rohr et al, J. Phys.: Conf. Series 664, 082047 (2015)

[4] D. Rohr et al, J. Phys.: Conf. Series 898, 032030 (2017)

[5] D. Rohr et al, these proceedings (2020)

[6] L. Evans et al, JINST 3 S08001 (2008) 
[7] CMS Collaboration, JINST 3 S08004 (2008)

[8] A. Bocci, these proceedings (2020)

[9] LHCb Collaboration, JINST 3 S08005 (2008)

[10] D. H. Cámpora Pérez, these proceedings (2020)

[11] C. D. Jones et al, J. Phys.: Conf. Series 513, 022034 (2014)

[12] C. D. Jones et al, J. Phys.: Conf. Series 664, 072026 (2015)

[13] C. D. Jones et al, J. Phys.: Conf. Series 898, 042008 (2017)

[14] C. D. Jones, presented in CHEP 2018, CMS-CR-2018-277 (2018)

[15] D. Dagenhart et al, these proceedings (2020)

[16] Threading Building Blocks, https://gi thub.com/intel/tbb

[17] CUDA C Programming manual

[18] CUB, https://nvlabs.github.io/cub/ 